DOI 10.31489/2021No2/60-66

UDC 621.43

\title{
THERMOACOUSTIC ENGINE AS A LOW-POWER COGENERATION ENERGY SOURCE FOR AUTONOMOUS CONSUMER POWER SUPPLY
}

\author{
Mekhtiyev A.D. ${ }^{1,2}$, Sarsikeyev Y.Z. ${ }^{1}$, Yugay V.V. ${ }^{3}$, Neshina Y.G. ${ }^{3}$, Alkina A.D. ${ }^{3}$ \\ ${ }^{1}$ S.Seifullin Kazakh Agrotechnical University, Nur-Sultan, Kazakhstan \\ 2 National Research Tomsk Polytechnic University, Tomsk, Russia \\ ${ }^{3}$ Karaganda Technical University, Karaganda, Kazakhstan, 1 _neg@mail.ru
}

\begin{abstract}
The article deals with the issue of using a thermoacoustic engine as a low-power cogeneration source of energy for autonomous consumer power supply capable of operating on various types of fuel and wastes subject to combustion. The analysis of the world achievements in this field of energy has been carried out. A number of advantages make it very promising for developing energy sources capable of complex production of electrical and thermal energy with a greater efficiency than that of present day thermal power plants. The proposed scheme of a thermal power plant is based on the principle of a Stirling engine, but it uses the most efficient and promising thermoacoustic converter of heat into mechanical vibrations, which are then converted into electric current. The article contains a mathematical apparatus that explains the basic principles of the developed thermoacoustic engine. To determine the main parameters of the thermoacoustic engine, the methods of computer modeling in the DeltaEC environment have been used. A layout diagram of the laboratory sample of a thermal power plant has been proposed and the description of its design has been given. It has been proposed to use dry saturated steam as the working fluid, which makes it possible to increase the generated power of the thermoacoustic engine.
\end{abstract}

Keywords: cogeneration, energy, thermal power plant, thermoacoustic engine, alternative source, thermoacoustics.

\section{Introduction}

An urgent trend in the development of present day decentralized power engineering is the development of alternative energy sources of low power for consumer power supply remote from power centers [1]. The analysis of the literature [2-11] has shown that new designs of heat engines with external heat supply are being developed, which can be a cogeneration source of energy with the capacity of 1 to $100 \mathrm{~kW}$ for self-generation of energy at the place of its consumption without transport losses. The literature describes a number of successful projects for developing thermal power plants using engines with external heat supply (EEHS) for autonomous consumers capable of operating on various fuels, as well as waste prone to combustion, their basis is various modifications of EEHS operating according to the Stirling thermal cycle $[6,7,8]$. We can cite as an example the developments of the well-known world manufacturers, such as the Philips, STM Inc., the DaimierBenz, the Solo, the United Stirling, the Ecopower, the WhisperGen, the Microgen, the Lion-Powerblock, the EcoGenViessmann, etc. [1-5]. According to some economic and technical indicators, a Stirling engine (SE) is superior to internal combustion engines (ICE) and gas turbine plants (GTP), which makes it attractive for use in cogeneration energy sources of low power [4-11]. SE is capable of operating in cogeneration mode producing 5-8 $\mathrm{kW}$ of thermal energy per $1 \mathrm{~kW}$ of electrical energy depending on its design $[6,8]$. At the same time, the overall efficiency of cogeneration energy sources based on EEHS can reach up to $90 \%$ with simultaneous production of heat and electricity [4 - 8]. It should be noted the low cost of the produced energy and the long service life of the cogeneration source within 20 years [6 - 8].

The aim of the work is studying a thermoacoustic engine (TAE) operating according to the Stirling thermal cycle with the effect of a standing sound wave, as well as obtaining the dependences of acoustic and electrical power on the working medium pressure and changing pressure of the working medium depending on the heater temperature. Our task is to carry out studies of the thermoacoustic type EEHS [12-23] having a more simplified design in comparison with the classic Stirling engine. 
The scientific novelty lies in obtaining new dependences of the TAE parameters with structural differences from their counterparts.

\section{Theoretical part of the studies}

To calculate the parameters of TAE with a standing sound wave, which is formed when two traveling waves are superimposed [12-23], expressions 1-3 have been used. The condition is accepted that sound waves move towards the opposite axis along the conditional $x$ axis, while their frequency and amplitude are the same, $t=0$ and $\varphi_{1}-\varphi_{2}=0$.

$$
S=S_{1}+S_{2}=2 A \cdot \operatorname{coskx} \cdot \cos \omega \mathrm{t}=2 \mathrm{~A} \cos \frac{2 \pi x}{\lambda} \cos \omega \mathrm{t},
$$

where $k=\frac{2 \pi}{\lambda}$ is the wave number, $A$ is the amplitude, $\lambda$ is the wave length,

$$
\begin{aligned}
& S_{1}=A \cdot \cos (\omega t-k x), \\
& S_{2}=A \cdot \cos (\omega t+k x) .
\end{aligned}
$$

In the experiments there have been used resonators with the length of $500 \mathrm{~mm}$ to $1200 \mathrm{~mm}$; for each resonator length, its own vibration frequency corresponds, which can be determined by the well-known expression:

$$
\lambda=v / f,
$$

where $\lambda$ is the wave length, $v$ is the sound speed, $f$ is the fluctuations frequency.

The total acoustic power $P_{t o t}$ of the TAE can be presented by the expression:

$$
P_{t o t}=Q_{f i l}-Q_{a l}-\Delta Q
$$

where $\Delta Q$ is the heat loss in heat exchangers.

The acoustic power is directly dependent on temperature gradients, namely the difference in temperature of the working fluid in the "Heater" and "Cooler" systems. An important element of the TAE is a resonator, in which there resonates an acoustic wave caused by the heating of the working medium in a plate heat exchanger. The calculation of the resonator of the TAE studied can be performed by expressing its length as $L_{\text {res }}$, and its diameter as $d_{\text {res }}$. In this case, it is necessary to take into account the pressure fluctuation in the linear generator $\delta_{r}$, which is the TAE load, and the speed fluctuation $v_{r}$. These fluctuations at the entrance to the resonator are related to the pressure fluctuations in the resonator $\delta_{\text {res }}$ and the speed $v_{\text {res }}$ by the following relationship:

$$
\begin{aligned}
& \delta_{\text {res }}=\delta_{r} \cos \left(k L_{r e s}\right)-\sin \left(k l_{r e s}\right) 4 / \pi d^{2} \cdot v_{r} \rho v_{\text {sou }}, \\
& v_{\text {res }}=v_{r} \cos \left(k L_{\text {res }}\right)-\sin \left(k l_{\text {res }}\right) \delta_{r} \pi d^{2} / 4 \cdot \rho v_{\text {sou }},
\end{aligned}
$$

where $\rho$ is the working fluid density, $v_{\text {sou }}$ is the sound speed, $k$ is the wave number.

The vibration frequency of the magnet attached to the latex rubber membrane can be represented as $[12,15]$ :

$$
\omega=\sqrt{\delta g / m},
$$

where $\delta$ is the coefficient depending on the membrane material properties, $g$ is the membrane rigidity, $m$ is the magnet mass.

To estimate the energy parameters of TAE on a standing acoustic wave using theoretical information [12-19], we introduce a parameter associated with generation of acoustic power $W_{\text {res }}$ in the regenerator. The volumetric velocity $U_{v o l}(x)$ is determined taking into account the second-order acoustic power $V_{i} \quad$ passing through the conditional point $x$. Let us accept the condition that the linear velocity of the 
longitudinal movement of the working fluid (gas) in the volume of the regenerator $V$ can be represented as the dependence:

$$
U_{v o l}=d V / d t, i^{\circ} \quad 2 \pi \phi \rho \mathrm{Udt}=\omega / 2 \phi \rho \mathrm{Ud} .
$$

As a result, we obtain a standard expression associated with averaging over time by the gas flow, at which the work of the acoustic wave is performed [12-18]. The calculation of the thermal efficiency indicator can be performed by the expression:

$$
\eta=W_{r} / Q_{\text {fil }}
$$

where $W_{\text {res }}$ is the power applied to the linear generator.

$$
\eta_{\text {ef }}=W_{\text {el.out }} / Q_{\text {fil }},
$$

where $W_{\text {el.out }}$ is electrical power of the linear generator.

As a result, the efficiency of the TAE thermal Carnot cycle $[3,4]$ will be shorter and is determined by the expression:

$$
\begin{gathered}
\eta_{e f . \text { Karnot }}=\eta_{e f} / \eta_{\text {Karnot }}, \\
W_{r}=1 / 2 R_{v}\left[\delta_{r} v_{r e s}\right] .
\end{gathered}
$$

\section{Description of the object of study}

The object of study is a TAE operating according to the Stirling thermal cycle with the effect of a standing sound wave. A laboratory specimen of a thermal power plants (TPP) with an output electric power of $30 \mathrm{~W}$ based on TAE is shown in Figure 1.

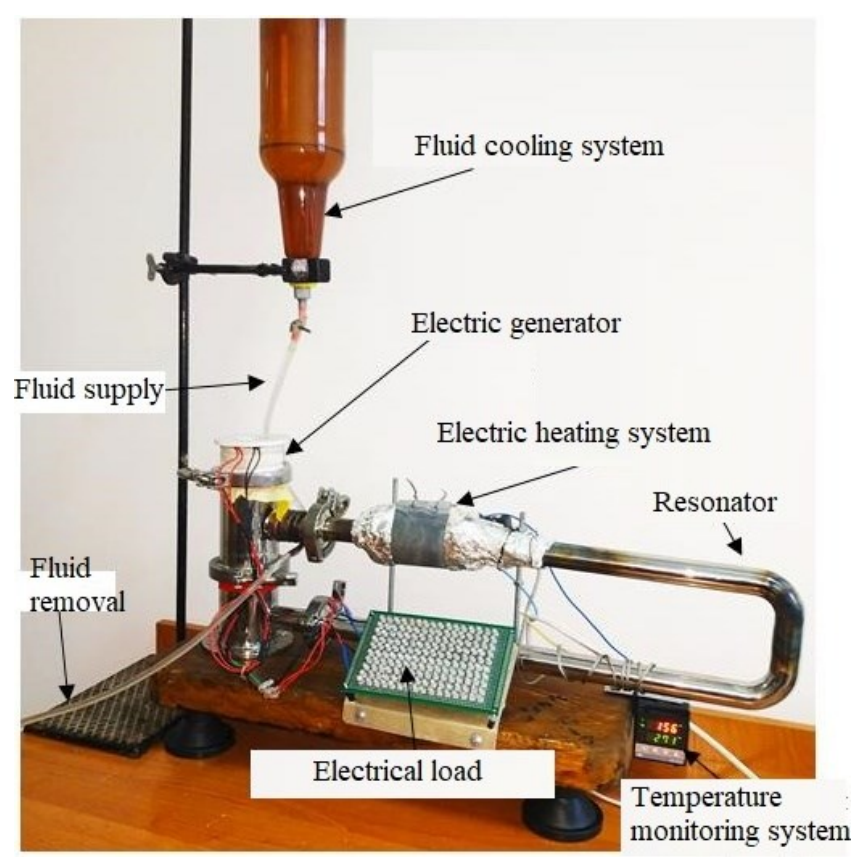

Fig.1. Laboratory TPP specimen base on TAE

Figure 2 shows a diagram of a laboratory specimen a TPP based on TAE. Heating of the working fluid is performed in plate heat exchanger 1 . Heating is performed using a $250 \mathrm{~W}$ electric spiral heater. In the experiment, air was used as the working medium with adding a small amount of water (vapor-air mixture), which provides the highest power indices of the studied laboratory TAD specimen. The gas pressure inside the resonator housing should be at least $0.15-0.2 \mathrm{MPa}$ [12-15]. The value of the initial pressure of the working fluid in the internal cavity of the TAE affects the value of its power. When heated, the gas volume expands and fills the entire cavity of the resonator, passes through regenerator 3 , which is made of a metal mesh with the cell diameter of about $1 \mathrm{~mm}$ [12-15]. Passing through, the heated 
gas gives up partially accumulated heat to the metal mesh of the regenerator; for the resonator tube diameter of 25 to $50 \mathrm{~mm}$, the length of the regenerator should be $50-80 \mathrm{~mm}$. The regenerator increases the thermal efficiency of the engine by $10-15 \%$, and reduces its heat losses. An important parameter is the developed temperature difference at the extreme regions of the regenerator, the greater it is, the greater its efficiency. Then, a slightly cooled working fluid enters the cold heat exchanger, where it is cooled and the process of its compression takes place. During cooling and compression, the working fluid (gas) begins to move in the opposite direction (indicated by the dotted line). Passing through the regenerator, the working fluid picks up partially accumulated heat by the regenerator and enters the heater already preheated.

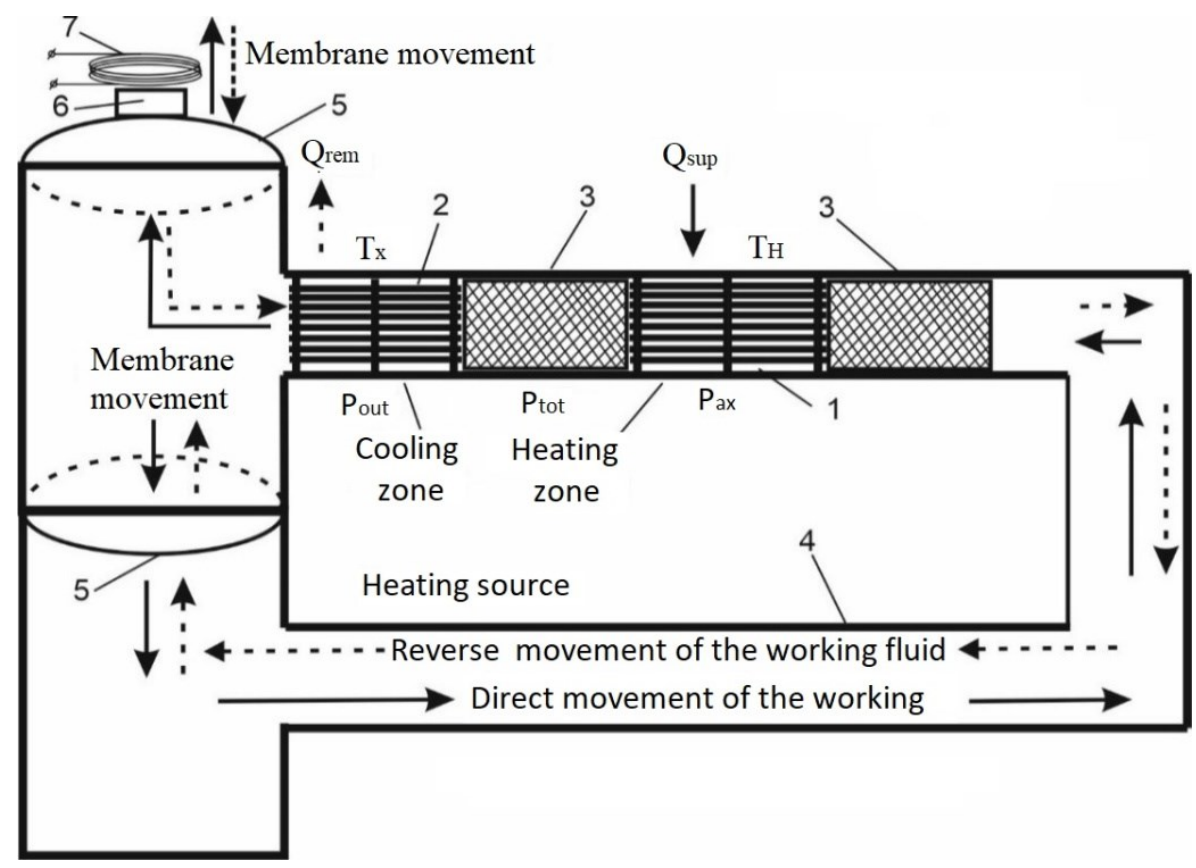

Fig.2. TAE based on the effect of a standing sound wave with an electrical and thermal circuit: 1 - hot heat exchanger, 2 - cold heat exchanger, 3 - regenerator, 4 - resonator, 5 - membrane, 6 - permanent magnet, 7 - coil with copper winding

When the working medium oscillates inside the resonator, its pressure and volume change, which activates membranes 5 , one of which is inside resonator 4 . When the working medium is heated, an acoustic wave is generated in the range of 150-180 dB, while the generation frequency depends on the length of resonator 4 and parameters of membrane rigidity 5 . The movement of gas inside the cavity of resonator 4 is shown in Figure 2, both ends of the resonator are closed by the tube with membranes 5, which are set in motion during the circulation of the working fluid. Permanent magnet 6 is fixed on the upper membrane, under the weight of which the membrane is lowered to the lower position, when the working fluid is compressed and cooled in cold heat exchanger 2, and when it expands, it rises. Magnet 6 reciprocates with the oscillation frequency of the resonator circuit. Magnet 6 moving up and down crosses coil 7 of the copper wire. An electrical load is connected to the output terminals of coil 7. The permanent magnet and the wound coil develop a linear electrical alternator. The use of the water circulation system in cold heat exchanger 2 improves the process of cooling the working fluid and increases the TAE power. Hot heat exchanger 1, cold heat exchanger 2 and regenerator 3 form the TAE working stage, the number of which can be increased to 4 , which will reduce the temperature gradient difference between the cold and hot parts of the stage up to 4 times.

\section{Experimental results}

There has been studied TAE with the resonator length of $1140 \mathrm{~mm}$ and the inner diameter of $22 \mathrm{~mm}$, operating with the use of the thermoacoustic effect of a standing acoustic wave in a heated gas. The oscillation frequency of the acoustic wave is within $300 \mathrm{~Hz}$, which is optimal for this type of the TAE 
design. With decreasing the length of the resonator, increasing the oscillation frequency of the rubber membrane is observed with decreasing the TAE power, and when using a $500 \mathrm{~mm}$ resonator, the oscillations of the membrane completely stopped. The air temperature in the room where the TAE tests have been carried out was $22-23{ }^{\circ} \mathrm{C}$. The dependence of changing pressure of the working medium on the temperature of the heater is shown in Figure 3. The efficiency of the TAE operation is largely affected by the temperature of the heater and cooler, as well as by pressure of the working medium in the resonator cavity. The working fluid pressure of the TAE has been measured when the temperature of the working fluid changed from $150^{\circ} \mathrm{C}$ to $330^{\circ} \mathrm{C}$. Increasing pressure and temperature allows increasing the power of the TAE, and reduces the time of self-start and the starting its operation. Figure 4 shows the dependence of the acoustic and electric power on the working fluid pressure that is the most important for estimating the TAE total efficiency.

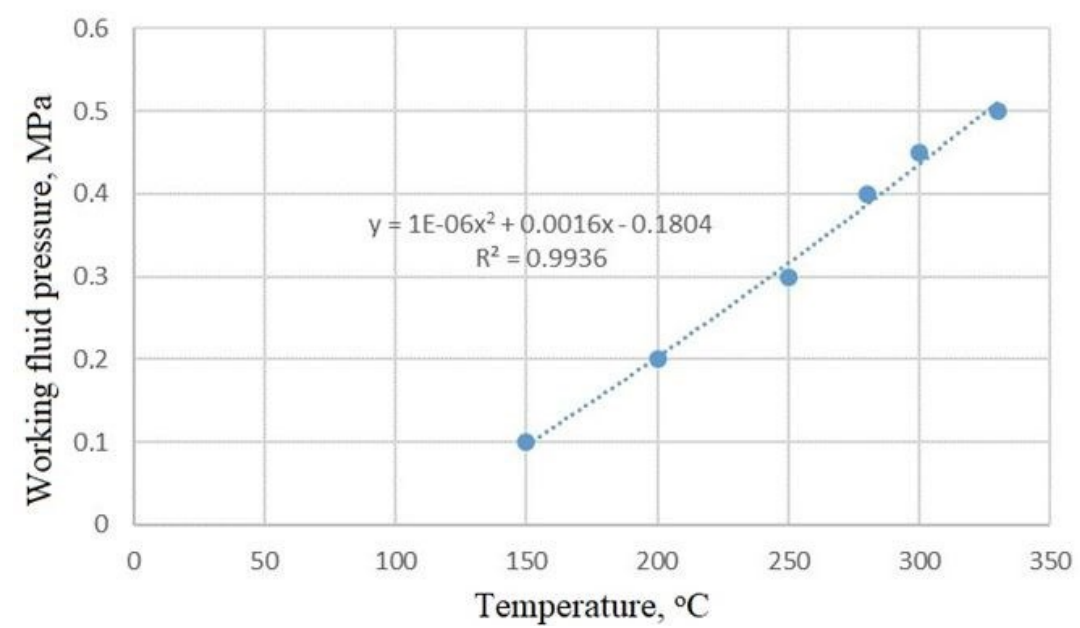

Fig.3. Dependence of the working fluid pressure changing on the heater temperature

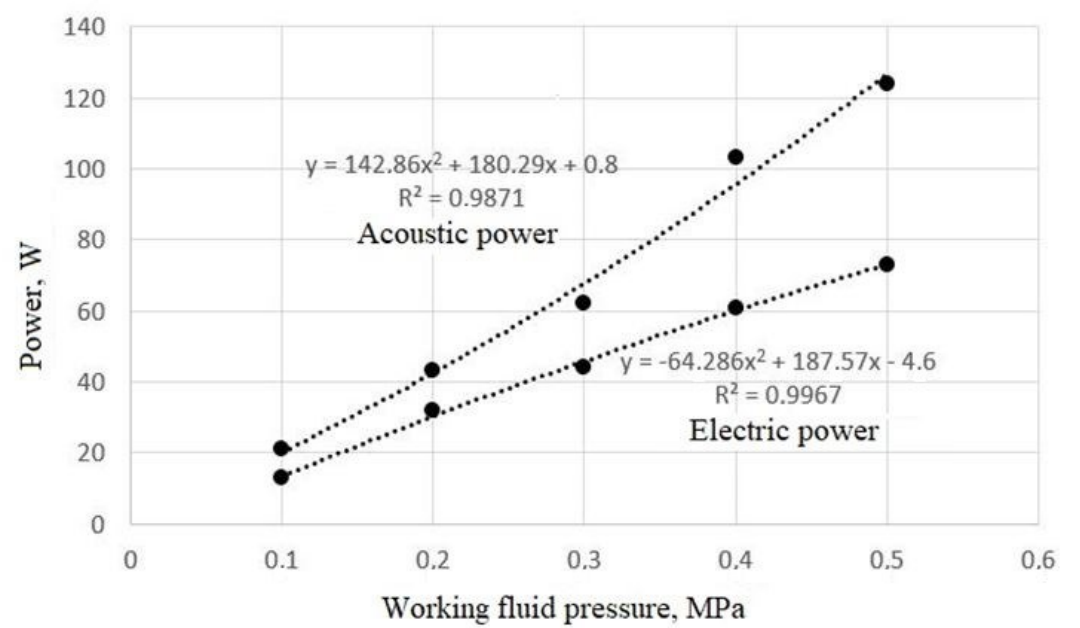

Fig.4. Dependence of the acoustic and electric power on the working fluid pressure

The design is intended for working pressure of $0.5 \mathrm{MPa}$; for operation at higher pressures in the range of 12-15 MPa, serious changes are required, first of all, the transition from a rubber membrane to a metal one which will also provide increasing the vibration frequency.

The DeltaEC computer program (Design Environment for Low-amplitude Thermo- Acoustic Energy Conversation developed by Los Alamos National Laboratory, USA, free license) has been used to calculate the parameters of the TPP laboratory specimen based on the TAE. This program is used to simulate thermoacoustic engines and to obtain their design parameters. DeltaEC performs numerical integration based on a low-amplitude linear approximation of the Rott equations. When comparing the results obtained experimentally and when modeling TAE with the use of the DeltaEC program, the values of the acoustic power of TAE were on average $30 \%$ higher than the experimental ones, since a lot of 
assumptions have been formed related to the external factors affecting the operation of TAE. The executed model in the DeltaEC program does not allow accurate reflecting all the design features and parameters of a full-scale specimen and has been used as a tool for preliminary calculation of the expected acoustic power. Subsequently, corrections will be made to this model to gradually approximate its parameters to a full-scale specimen due to the fact that the mechanism for developing heat exchangers and regenerators is insufficiently developed. There is also idealization of heat transfer processes and structural parts of the TAE, on the basis of which the model has satisfactory accuracy of information of the parameters and geometry (pressure, temperature, dimensions, operating frequency, etc.), but it does not take into account the hydrodynamic features of the flow and transient processes in various components of the pulsation cooler structure. Similar problems with the accuracy of modeling the software product DeltaEC are noted in works [21, 22]. In practical experiments with the laboratory TAE specimen, a microphone and Sound Card Oszilloscope software have been used to measure the amplitude of acoustic pressure in the resonator, which together with a computer performed the functions of a twochannel oscilloscope with the transmission frequency in the range from 20 to $20.000 \mathrm{~Hz}$.

\section{Conclusions}

The performed theoretical and practical studies allow us to assert that a single-stage TAE can be used as a low-power cogeneration energy source for power supply of autonomous consumers. TAE has a number of advantages, which makes it very promising for the creation of autonomous multi-fuel energy sources capable of more efficiently producing electric and thermal energy in a complex manner than modern thermal power plants (TPPs). The presented design of a single-stage TAE, based on the effect of a standing sound wave, ensures its launch at a temperature of about $150^{\circ}$, which is a rather significant indicator for a single-stage design. The use of an additional regenerator increases the thermal efficiency of the TAE. TAE operates according to the well-known Stirling thermal cycle, but unlike the known energy sources based on the Stirling engine, it does not have pistons and, accordingly, problems with ensuring the reliability of the seals, which makes it possible to increase its resource, as well as the overhaul interval. The use of the free software product DeltaEC made it possible to calculate the TAE parameters and simulate its operation, which reduced the time spent on its creation.

The minimum indicator of the TAE self-start temperature of $186^{\circ} \mathrm{C}$ with the load of $30 \mathrm{~W}$ was reached with the resonator with the inner diameter of $22 \mathrm{~mm}$ and the length of $1140 \mathrm{~mm}$. The proposed air-vapor mixture as a working fluid is capable of effectively operating in the resonator with the length of $1140 \mathrm{~mm}$ at the minimum $\mathrm{T}=150{ }^{\circ} \mathrm{C}$ with pressure $\mathrm{P}=0.1 \mathrm{MPa}$ and at the maximum $\mathrm{T}=330{ }^{\circ} \mathrm{C}$ with pressure $\mathrm{P}=0.5 \mathrm{MPa}$. Increasing the density of the working fluid is a very promising direction for increasing pressure at average temperatures of the working fluid from $150^{\circ} \mathrm{C}$ to $330^{\circ} \mathrm{C}$, which is not achievable when used in air as a working fluid. This circumstance makes it possible to significantly increase the power of the TAE and create a commercially attractive cogeneration autonomous energy source capable of operating on almost any type of fuel, including waste that is subject to combustion, since only a heat source is needed for its operation.

\section{Acknowledgments}

The article was prepared within the framework of the project AP05131751 "Micro thermal power plant of the cogeneration type with heat recovery".

\section{REFERENCES}

1 Mekhtiyev A.D., Yugay V.V., Alkina A.D., Kaliaskarov N.B., Esenzholov U.S. Multi-fuel micro-thermal power plant with a capacity of 1-10 kW for remote facilities in rural areas and farms. Bulletin of the South Ural State University, 2018, Vol. 18, No. 2, pp. 62-71. [in Russian]

2 Mekhtiyev A.D., Yugay V.V., A.D.Alkina, N.B.Kaliaskarov, U.S. Esenzholov. Multi-fuel power plant with a heat engine External combustion, able to work effectively in the conditions of rural Locations of kazakhstan. International research journal, 2018, No.12 (78), pp. 124-131.

3 Mekhtiev A.D., Yugay V.V., Esenzholov U.S., Kaliaskarov N.B. Engine with external heat supply based on thermoacoustic effect for autonomous thermal power plant. Bulletin of the South Ural State University. Series: Energy, 2019, No. 2, pp. 22-30. [in Russian] 
4 Mekhtiyev A.D., Yugay V.V., Esenzholov U.S., Mekhtiyev R.A. Prospects for the use of ultra-low-power thermal power plants based on an engine with an external heat supply. Mechanics and technologies, 2019, No. 2 (64), pp. 166-173. [in Russian]

5 Mekhtiev A.D., Yurchenko A.V., Yugay V.V., Al'kina A.D., Yessenzholov U.S. Multi-fuel power station of ultra-low power with external combustion thermal engine, capable efficiently operate in the conditions of rural areas of Kazakhstan. News of the national academy of sciences of the republic of Kazakhstan series of geology and technical sciences, 2019, Vol.3, No.435, pp.136 - 143.

6 Reeder G. Stirling Engines. Moscow, Mir,1986, 464 p. [in Russian]

7 Walker G. Stirling Engines. Moscow, Mechanical engineering, 1985, 408 p. [in Russian]

8 https://ru.wikipedia.org/wiki

9 Makushev Yu.P., Polyakova T.A., Ryndin V.V., Litvinov P.V. Methods for calculating the operating cycle of a petrol engine with construction of the indicator diagram. Science and technology of Kazakhstan, 2018, No. 2, pp. 63-81. [in Russian]

10 Volkova A.V., Ryzhenkova A.V., Parygina A.G., Naumova A.V., Druzhinina A.A. Matters Concerned with Development of Autonomous Cogeneration Energy Complexes on the Basis of Microhydropower Plants. Heat power engineering, 2018, No. 11, pp. 32-39. [in Russian]

11 Efendiev A.M., Nikolaev Yu.E., Evstafiev D.P. Possibilities of power supply of farms based on small renewable energy sources. Heat power engineering, 2016, No. 2, pp. 38-45. [in Russian]

12 Zinoviev E.A., Dovgyallo A.I. To assessment of efficiency indicators of the working process of the thermoacoustic engine. Bulletin of the Samara State Aerospace University, 2012, No.3 (34), pp. 145-152. [in Russian]

13 Hamood A., Jaworski A.J., Mao X., Simpson K. Design and construction of a two-stage thermoacoustic electricity generator with push-pull linear alternator. Energy, 2018, No.144, pp. $61-72$.

14 Jin T., Yang R., Wang Y., Feng Y., Tang K. Low temperature difference thermoacoustic prime mover with asymmetric multi-stage loop configuration. Sci. Rep. UK, 2017, Vol.7, pp. 1-8.

15 Ceperley P. H. A pistonless Stirling engine - the traveling wave heat engine. J. Acoust. Soc. Am, 1979, Vol. 66, No.5, pp. 1508-1513.

16 Piccolo A., Pistone G. Estimation of heat transfer coefficients in oscillating flows: The thermoacoustic case. Int. J. Heat Mass Tran, 2006, Vol. 49, pp. 1631-1642.

17 Abduljalil A. S., Yu Z., Jaworski A. J. Mater. Design, 2011, Vol. 32, pp. 217-228.

18 Langlois L., Justin R. Dynamic computer model of a Stirling space nuclear power system / Justin L.R. Langlois. Trident Scholar project report, 2006, No. 345, pp. 348 - 352.

19 Wang K., Qiu L. Numerical analysis on a four-stage looped thermoacoustic Stirling power generator for low temperature waste heat. Energ. Convers. Manage, 2017, Vol. 150, pp. 830-837.

20 Piccolo A., Pistone G. Estimation of heat transfer coefficients in oscillating flows: The thermoacoustic case. Int. J. Heat Mass Tran, 2006, Vol. 49, pp. 1631-1642.

21 Nekrasova S.O., Sarmin D.V., Uglanov D.A., Shimanov A.A. Numerical and experimental study of thermoacoustic cooler on a pulse pipe. Bulletin of the Samara Scientific Center of the Russian Academy of Sciences, 2015, Vol. 17, No.6 (2), pp.125-135. [in Russian]

22 Gorshkov I.B., Izv V.V. Numerical simulation of a ring four-stage thermoacoustic running wave engine. Saratov Univ. (N. S.), Ser. Physics, 2018, Vol. 18, No. 4, pp. 285-296. [in Russian] 